\title{
Evaluation of a Genetic Representation for Outline Shapes
}

\author{
P. Lapok \\ Edinburgh Napier University \\ EH10 5DT \\ United Kingdom \\ p.lapok@napier.ac.uk
}

\author{
A. Lawson \\ Edinburgh Napier University \\ EH10 5DT \\ United Kingdom \\ a.lawson@napier.ac.uk
}

\author{
B. Paechter \\ Edinburgh Napier University \\ EH10 5DT \\ United Kingdom \\ b.paechter@napier.ac.uk
}

\begin{abstract}
This work in progress focuses on the evaluation of a genetic representation for outline shapes for planar mechanical levers which addresses the first stage of the complex real-world problem of modelling and evolving planar mechanical lever systems. The representation defines the genotype and the phenotype mapping onto a lever geometry. As a proof of concept, the representation was evaluated using an evolutionary algorithm with simplified fitness function to investigate its capability to facilitate search space coverage, work with evolutionary operators; and produce shapes with certain lever characteristics, such as edges and curves.
\end{abstract}

\section{KEYWORDS}

Shape Representation, Genetic Representation, Evolutionary Algorithm, Mechanical Lever Systems, Complex Systems

\section{INTRODUCTION}

The design of planar mechanical lever systems can be a complex task for human designers, e.g. the design of locking mechanisms in the automotive industry. The design task is to produce a system of levers that will fulfil a specified behavior in terms of component movements and force transmission, within a constrained dynamic spatial environment. A planar lever system can be described in terms of the shape and position of mechanical components within the system, along with their kinetic and kinematic properties. The behaviors of lever systems are determined by their individual outline shapes, position constraints, and outline interactions.

This work in progress focuses on the sub-problem of defining a suitable representation for the 2-dimensional outline shapes of

Permission to make digital or hard copies of all or part of this work for personal or classroom use is granted without fee provided that copies are not made or distributed for profit or commercial advantage and that copies bear this notice and the full citation on the first page. Copyrights for components of this work owned by others than ACM must be honored. Abstracting with credit is permitted. To copy otherwise, or republish, to post on servers or to redistribute to lists, requires prior specific permission and/or a fee. Request permissions from Permissions@acm.org.

GECCO '17 Companion, July 15-19, 2017, Berlin, Germany

(c) 2017 Association for Computing Machinery.

ACM ISBN 978-1-4503-4939-0/17/07...\$15.00

http://dx.doi.org/10.1145/3067695.3082501 levers as a first step toward solving the larger problem of how to evolve planar mechanical lever systems. Previous work using shape representations in the mechanical engineering domain includes topology optimization. In this work, various optimization techniques are applied to improve the structure of a mechanical part by reducing material usage whilst keeping its stability [1-8]. Cellular representations such as pixels or voxels were used. Pixel representations produce 2-dimensional shapes and voxel representations produce 3-Dimensional shapes. These has also been used in other areas such as evolving soft robots [9].

Other previous work has investigated the evolution and optimization of conceptual designs in the mechanical engineering domain [10-13]. These are mostly task specific representations which parameterized characteristics of objects or specific fixed components.

In this work, a new representation for outline shapes is proposed for use in an evolutionary computing context. A methodology for the evaluation and comparison of outline shape representations is presented. The distinctive characteristics of a suitable representation are investigated, namely: search space coverage; compatibility with evolutionary operators; and genotype to phenotype mapping. A suitable representation is developed using an iterative process of experimentation and evaluation. This includes the development of a fitness function for evaluating the shapes produced by the representation against a defined range of target shapes. This work is a first step towards generating planar mechanical lever systems using an evolutionary computing approach.

\section{REPRESENTATION SPECIFICATION}

\subsection{Search Space Coverage}

One benefit of an evolutionary approach might be that novel designs could be found that might not have been thought of by human designers. This initial work is not interested in evolving novel shapes or every possible kind of shape. The focus is to develop a process for evaluating representations which can evolve different shape characteristics for levers within a mechanical engineering context. In the future, the representation will be extended to investigate the generation of novel mechanical system designs consisting of a set of levers. These characteristics include: corners; curves; symmetries; and asymmetries. A set of target shapes was defined to allow evaluation of the representation's ability to cover the search space of characteristic shapes. These shapes may of themselves 
not be valid lever designs, but have been chosen to allow the evaluation of the representation based on its ability to represent distinctive characteristics of lever shapes. The target shapes are shown in Fig. 1.

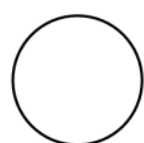

(a) circle

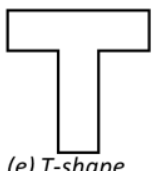

(e) T-shape

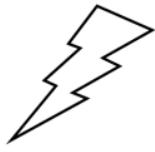

(i) flash

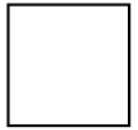

(b) square
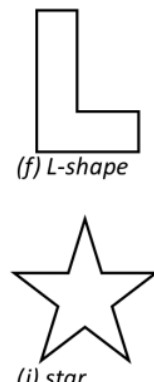

(j) star

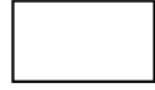

(c) rectangle

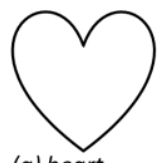

(g) heart

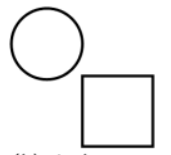

(k) circle-square

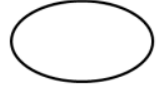

(d) ellipsis

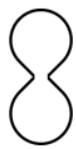

(h) time-glass

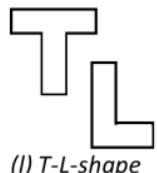

Figure 1: Range of target shapes

\subsection{Compatibility with Evolutionary Operators}

The genetic representation needs to be compatible with the operators used in an evolutionary algorithm. For mutation operators, it is necessary that a changes in the genotype results in equal sized changes in the phenotype [14]. For recombination operators, it is necessary that some characteristics of each of the parent phenotypes be passed to the child phenotype.

As an example, a phenotype could be defined by an origin coordinate and a set of vertex coordinates, describing the shape. A mutation operator should produce a small manipulation such as the change of position of a single vertex or of a small set of vertices on the phenotype. A recombination operation, such as one-point crossover should produce a swap of a set of vertices between two parent phenotypes which would result in child solutions such as shown in Fig. 2.

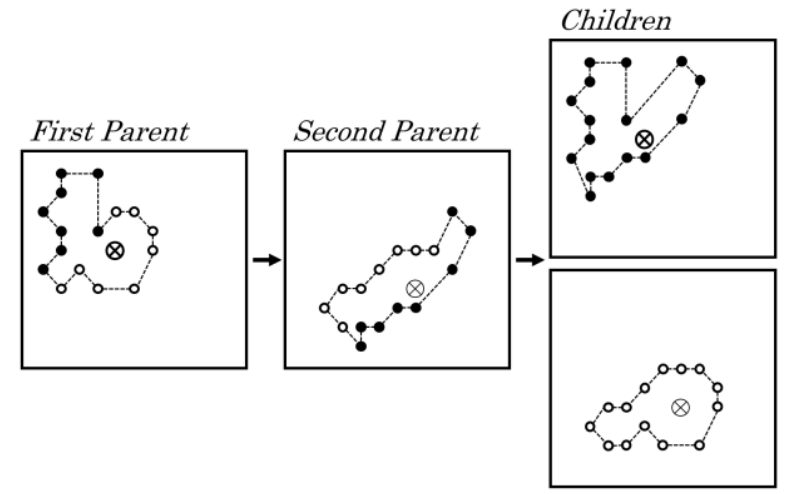

Figure 2: The recombination of two parent solutions into two potential child solutions by recombination of vertices and position of origin.

\subsection{Genotype to Phenotype Mapping}

From the perspective of the genotype, the size of the chromosome is defined by the number of genes needed to represent a solution. The chromosome size has a direct influence on the size of the search space. The larger the search space, the more the number of iterations required to traverse the search space. Therefore, a genotype with a small number of genes is preferred, especially as the shape representation will be only one part of a future representation for planar lever systems which will require more complexity in terms of the number of parameters to include aspects related to kinematics.

From the perspective of the phenotype, the representation should not result in invalid outline shapes. Invalid shapes are self-intersecting shapes. Fig. 3 shows (a) a valid shape and (b) an invalid self-intersecting shape.
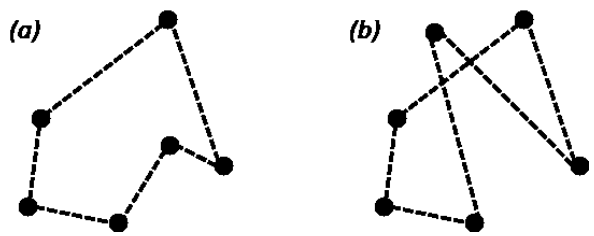

Figure 3: Example of shapes: (a) Valid shape, (b) Invalid shape

Using a representation with a direct mapping of coordinates to vertex position in a Cartesian coordinate system would introduce the possibility of producing many invalid selfintersecting shapes. A representation which allows such selfintersecting shapes would require an additional layer of computation to filter or to repair invalid shapes. It also increases the search-space as the number of invalid shapes is larger than the number of valid shapes, which means that many chromosomes would produce no valid solutions. A better representation would be one which avoids the possibility of these self-intersections.

\section{METHODOLOGY}

\subsection{Representation}

The behavior of a rigid body lever system results from the contacts of outline shapes and their interaction without considering the structure of the surface area of each individual component. One disadvantage of using a cellular representation is the amount of information that needs to be used to describe the structure, which in turn increase the search space to be traversed. A new representation was designed and evaluated. This representation produces only non-intersecting shapes. The chromosome consists of relative float values between 0.01 and 1.00 which are converted to angles, and lengths through a mapping process. The representation $R$ is shown in Fig. 4. 


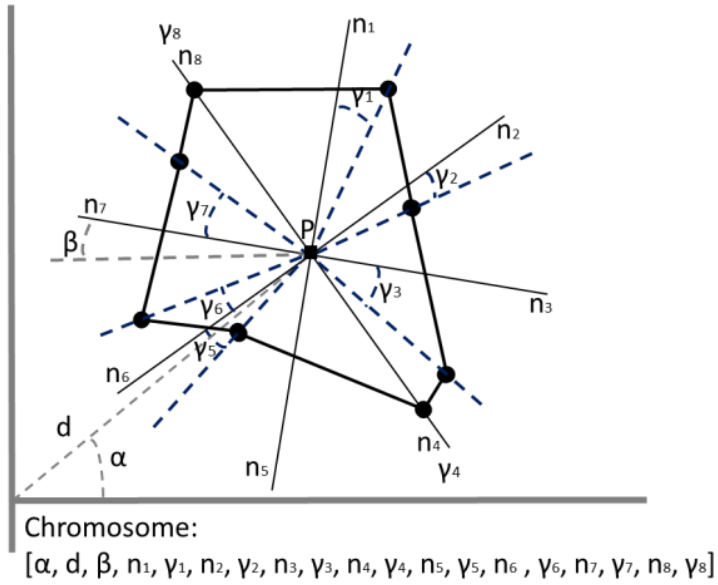

Figure 4: Representation $\boldsymbol{R}$

The representation consists of a movable hub and spoke configuration with the origin $P$, placed within a polar coordinate system. $P$ is defined by two location genomes, $\alpha$ and $d$. The hub and spoke configuration has a variable number of arms, each holding one vertex. The number of arms is defined by the user with a global parameter. The angles between the arms are equal and results from the number of arms used. The position of a vertex on an arm is defined by a genome $n$ which represent the distance between $P$ and the vertex itself. The maximum distance between $P$ and a vertex is also specified by the user with a global parameter. There are two additional angles $\beta$ and $\gamma . \beta$ rotates the hub and spoke configuration around its origin $P$, and $\gamma$ is an angle between each arm. $\gamma$ allows each arm and its containing vertex to be rotated between the initial arm position and the initial position of the next arm.

\subsection{Evolutionary Algorithm}

The representation was tested using a standard evolutionary algorithm to evaluate its ability to produce a set of defined target shapes with distinctive characteristics and complexity. The evolutionary algorithm was configured as follows: The population was set to 100 individuals and 10 children were produced per generation. These parameters were chosen after some initial tests. Elite-tournament with two random individuals taken from the population was used to pick the fitter candidates for either a two-point crossover operation or a simple mutation operation with a chance of $50 \%$ for each operation in every iteration. Crossover and mutation were used to investigate the behavior of the representation when applying genetic operators. The simple mutation operator changed a random number of genomes from one genome up to $1 / 4$ of the length of the chromosome by changing it to a random float value. The children were placed back into the population by using a riptournament between two random candidates from the population to replace the weaker one.

\subsection{Fitness Evaluation}

To test the representation's ability to evolve target shapes, a simplified fitness function was developed. The fitness function is based on two penalty values related to the surface area of the shapes. Fig. 5 shows the equation for the fitness.
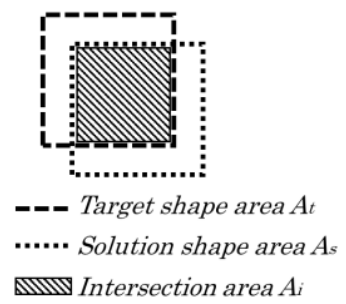

$$
\begin{aligned}
& \text { (a) Size penalty } P_{s} \\
& P_{s}=\left|A_{s}-A_{t}\right|
\end{aligned}
$$

(b) Intersection penalty $P_{i}$

$$
P_{i}=|A t-A i|
$$

(c) Total penalty $P_{t}$

$P_{t}=P_{s}+P_{i}$

\section{Figure 5: Fitness Evaluation}

A lower penalty value represents a fitter solution. The first penalty value is the size-penalty $(a)$. It describes the difference in surface area between the candidate solution and target shape. E.g. their position does not need to be the same but if their size is equal, the penalty would be zero. The second penalty is the intersection-penalty $(b)$ which is given related to the intersection between solution and target. A solution which over-covers the target area would result in a penalty of zero. The total penalty (c) is a sum of both penalty values. A smaller total penalty value stands for a better solution. A total penalty value of zero means that the solution and the target shape are identical in shape and position.

\section{PROOF OF CONCEPT EXPERIMENTS}

Experiments were run several times on each target shape, for both 8 vertices per shape, and for 16 vertices per shape. Each experiment was stopped after 500,000 iterations. The best results are shown in Fig. 6 as a proof of concept that the representation can approximate the target shapes. Fig. 6 shows the best solutions from the experiments with 8 and 16 vertices. The number of vertices used is noted next to the shapes name. The evaluation of the efficiency of the algorithm and representation was not considered for this proof of concept work, but will be investigated in future.

It was found that the representation can approximate the target shapes with different shape characteristics for shapes (a) to $(j)$. The representation only produces valid, non-selfintersecting shapes e.g. as discussed in section 3.3. The results show that the representation can be used with evolutionary techniques, e.g. mutation and crossover operators to navigate through the search space. 

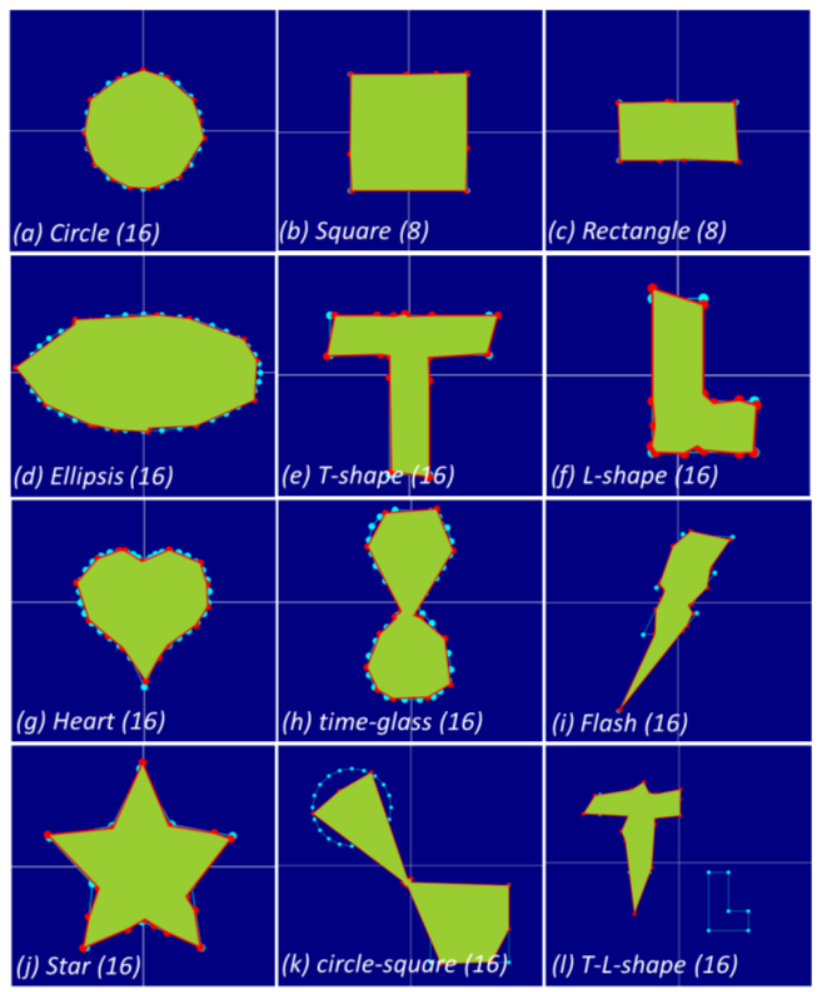

Figure 6: Resulting Shapes

It was also found that a higher number of vertices lead in most cases to a better result especially if the target shape is more complex because it allows the representation to describe it with a higher level of detail. However, the algorithm may get stuck with a slightly disrupted shape when using a high number of vertices, e.g. in case of simple shapes such as square (b) or rectangle (c). This is due to the smaller chance of organizing multiple vertices on a straight line. For these shapes a lower number of vertices produce better results. Curved shapes, such as heart, time-glass, and more complex shapes, such as flash and star, need at least 16 vertices to be described accurately. In future, the representation should be extended with a mechanism which controls the number of vertices during the search process, starting with a lower number of vertices at the beginning of the search process.

The representation in its current state is not capable of representing unconnected shapes $(k)$ and $(l)$. This will be addressed in future by allowing the representation to adapt by generating multiple sub-shapes.

\section{CONCLUSIONS}

This work in progress proposed a representation for outline shapes for levers, with a view to future extension and use as a representation for evolving planar mechanical lever systems. A characteristic of the representation is that it avoids the production of self-intersecting shapes which reduces the search space. The representation's capability to evolve and represent specified target shapes with distinctive characteristics was tested using an evolutionary algorithm based on a fitness function comparing the geometrical areas of candidate shape and a target shape. The results show that the proposed representation can produce the defined shape characteristics for a set of single shapes. Results also show that the representation is compatible with evolutionary computing techniques for traversing the search space. It was found that additional mechanisms are needed to make the representation more accurate. Firstly, a mechanism to control the number of vertices allowing a decrease or increase of the level of representation detail, and the secondly, a mechanism to allow the solution shape to be divided into multiple sub-shapes to represent unconnected shapes. Subshapes may also increase the accuracy for describing target shapes made of multiple primitives.

Future work will implement the above mechanisms and will evaluate the efficiency of the representation using statistical analysis. Furthermore, the representation will be extended to evolve planar mechanical lever systems.

\section{REFERENCES}

[1] Chapman, C. D., \& Jakiela, M. J. (1996). Genetic algorithm-based structural topology design with compliance and topology simplification considerations. Fournal of Mechanical Design, 118(1), 89-98.

[2] Chen, S. Y., \& Rajan, S. D. (2000). A robust genetic algorithm for structural optimization. Structural Engineering and Mechanics, 10(4), 313-336.

[3] Duda JW, Jakiela MJ. Generation and Classification of Structural Topologies With Genetic Algorithm Speciation. ASME. J. Mech. Des. 1997;119(1):127131.

[4] Baron, P., Fisher, R., Tuson, A., Mill, F., \& Sherlock, A. (1999). A voxel-based representation for evolutionary shape optimization. AIEDAM, 13(3), 145156.

[5] Eby, D., Averill, R. C., Punch, W. F., \& Goodman, E. D. (1999). Optimal design of flywheels using an injection island genetic algorithm. AI EDAM, 13(05), 327-340.

[6] Smith, R., Warrinton, S., \& Mill, F. (1995, September). Shape representation for optimisation. In Genetic Algorithms in Engineering Systems: Innovations and Applications, 1995. GALESIA. First International Conference on (Conf. Publ. No. 414) (pp. 112-117). IET.

[7] Deb, K. (1997). GeneAS: A robust optimal design technique for mechanical component design. In Evolutionary algorithms in engineering applications (pp. 497-514). Springer Berlin Heidelberg.

[8] Grierson, D. E., \& Pak, W. H. (1993). Optimal sizing, geometrical and topological design using a genetic algorithm. Structural Optimization, 6(3), 151-159.

[9] Cheney, N., MacCurdy, R., Clune, J., \& Lipson, H. (2013, July). Unshackling evolution: evolving soft robots with multiple materials and a powerful generative encoding. In Proceedings of the 15th annual conference on Genetic and evolutionary computation (pp. 167-174). ACM.

[10] Bentley, P. J., \& Wakefield, J. P. (1997). Conceptual evolutionary design by a genetic algorithm. Engineering design and automation, 3, 119-132.

[11] Cvetkovic, D., \& Parmee, I. C. (1999, August). Genetic algorithms based systems for conceptual engineering design. In Proceedings of the 12th International Conference on Engineering Design ICED (Vol. 99, pp. 1035-1038).

[12] Rasheed, K., Hirsh, H., \& Gelsey, A. (1997). A genetic algorithm for continuous design space search. Artificial Intelligence in Engineering, 11(3), 295-305.

[13] Choi, B. G., \& Yang, B. S. (2000). Optimum shape design of rotor shafts using genetic algorithm. Fournal of Vibration and Control, 6(2), 207-222.

[14] Bentley, P. (1999). Evolutionary design by computers. Morgan Kaufmann. 